\title{
High Capacity Multi-Mode Transmission Systems Using Higher-Order Modulation Formats
}

\author{
V.A.J.M. Sleiffer*, Y. Jung**, P. Leoni***, M. Kuschnerov****, R.G.H. van Uden*, \\ V. Veljanovski****, L. Grüner-Nielsen*****, Y. Sun*****, D. Richardson**, S. U. Alam**, \\ F. Poletti**, B. Corbett******, R. Winfield $* * * * * *$ and H. de Waardt* \\ * COBRA institute, Eindhoven University of Technology, Eindhoven, The Netherlands \\ ** Optoelectronics Research Centre, University of Southampton, Southampton, SO17 1BJ, UK \\ *** Universität der Bundeswehr München, 85577 Neubiberg, Germany \\ **** Nokia Siemens Networks Optical GmbH, St-Martin-Str. 76, Munich, Germany \\ ***** OFS, Priorparken 680, 2605 Brøndby, Denmark \\ ****** Tyndall National Institute, Cork, Ireland
}

\begin{abstract}
We look at multi-mode fiber as potential means to upgrade capacity of optical transmission systems compared to current single-mode technology by employing multiple modes as transmission lanes as well as using higher-order modulation formats.
\end{abstract}

\section{INTRODUCTION}

In the next 10 to 20 years the current deployed optical long-haul transmission systems will be fully lit due to the $\sim 40 \%$ annual increase in data traffic and operators will have to install new fiber. Typically this is the most costly part of expanding the capacity of their systems. The preferred way to increase capacity is moving to higher order modulation formats and/or multiple carriers to increase the spectral efficiency (SE), e.g. from the currently being deployed $100-\mathrm{Gb} / \mathrm{s}$ Dual-Polarization (DP) Quadrature Phase-Shift Keyed (QPSK), to 400-Gb/s dual-carrier DP-16-level Quadrature Amplitude Modulation (16QAM) [1,2]. The move to higher-order modulation formats will require more received OSNR for the same performance at the same line-rate. Therefore further increasing SE will push system requirements to very high received OSNRs, especially due to additional implementation penalties. The launch power cannot be increased due to single-mode fiber nonlinear impairments. The two effects combined, i.e. received OSNR and nonlinear impairments, therefore put a hard limit on the maximal SE that can be obtained for a certain transmission distance [3]. And research already approached that single-mode fiber (SMF) SE limit [4]. Therefore sooner or later new fibers will have to be deployed and then it would be the most beneficial if the new fibers offer more capacity to avoid, or at least delay, a capacity crunch and offer the possibility to integrate components to save space, energy and money.

Multi-mode fiber is considered to be one of the possible solutions to offer more capacity per fiber. In the past multi-mode fibers were not used because of intermodal dispersion which limited the transmission distance to several hundreds of meters. But with the advance of coherent technology and especially digital signal processing (DSP), new opportunities have arisen. Several space-division-multiplex (SDM) experiments [5-8] have been successfully conducted, using special few-mode fibers (FMFs), which typically contain less than 10

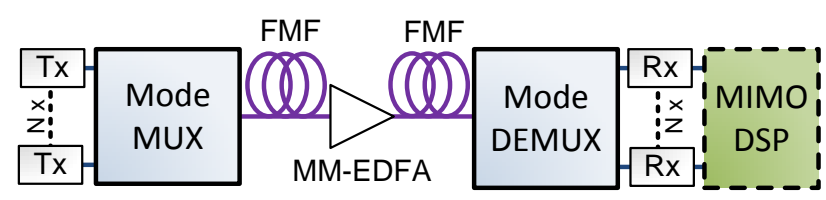

Fig. 1. FMF Transmission consisting of $\mathrm{N}$ transmitters, a mode multiplexer (MUX), N mode supporting few-mode fiber (FMF), a multimode EDFA (MM-EDFA), mode de-multiplexer (DEMUX), $\mathrm{N}$ coherent receivers and MIMO-DSP.

modes (as compared to conventional multi-mode fibers having >100 modes), and multiple-input multiple-output (MIMO)-DSP.

In this paper we will discuss the possibility to increase the capacity by the use of FMF and higher-order modulation formats and have a look at a potentially promising fiber type, the photonic bandgap fiber (PBGF).

\section{HIGH-CAPACITY FEW-MODE FIBER TRANSMISSION SYSTEMS}

By using FMF the capacity per fiber can be increased by using the modes as separate transmission lanes. The capacity per fiber is directly increased by a factor equal to the number of supported modes in the fiber. To exploit the modes in FMF systems $\mathrm{N}$ single-mode signals, where $\mathrm{N}$ equals the number of modes of the FMF, have to be mode-multiplexed (Mode MUX) at the transmitter side, and de-multiplexed (Mode DEMUX) at the receiver side, therefore requiring $\mathrm{N}$ transmitters and $\mathrm{N}$ coherent receivers (Fig. 1). A key component for FMF systems to become viable is the multi-mode (MM) amplifier. Using such a MM- erbium-doped fiber amplifier (EDFA) mid-span successful transmission of 3 modes (the linearpolarized $\mathrm{LP}_{01}$ and degenerate $\mathrm{LP}_{11 \mathrm{a}}$ and $\mathrm{LP}_{11 \mathrm{~b}}$ modes) carrying 100-Gb/s DP-QPSK over FMF was shown with

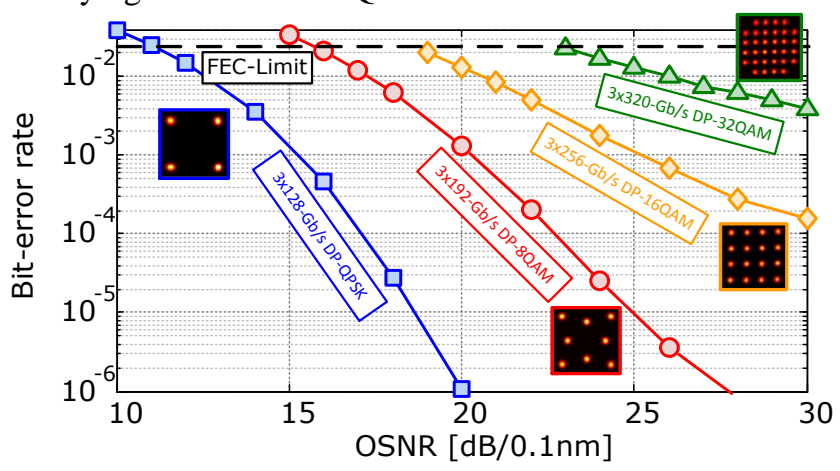

Fig. 2. Measured average back-to-back curves for three multiplexed modes 
TABLE I

MULTI-MODE SOLID CORE FIBER AND MULTI-MODE HOLLOW CORE COMPARED WITH SINGLE-MODE FIBER

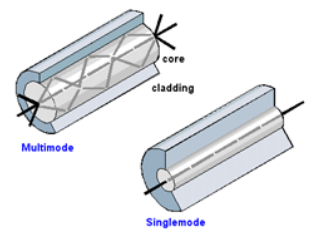

Multi-mode solid core

$\checkmark$ Record capacity so far $73.7 \mathrm{~Tb} / \mathrm{s}$

$\checkmark$ Enabling higher density of integration for transponders \& amplifiers, ROADMs

$\checkmark$ Higher reach due to lower nonlinearity

$\checkmark$ More efficient pumping than multi-core

- Same bandwidth available as SMF

Equalization of mode crosstalk required in DSP

Mode dependent loss can lead to performance degradation

Scalability to higher number of modes is tough for the fiber design

- Same loss as SMF

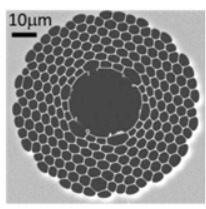

Multi-mode hollow core

$\checkmark$ Lowest latency ( $\sim 97 \%$ speed of light)

$\checkmark$ Enabling higher density of integration for transponders \& amplifiers

$\checkmark$ Lowest loss $(<0.1 \mathrm{~dB} / \mathrm{km}) @ 2 \mu \mathrm{m}$

$\checkmark$ Very low nonlinearity

$\checkmark 4 \times$ Bandwidth available of SMF @ 2um

Very high differential group delay requires huge DSP complexity

Currently the intrinsic mode dependent loss is high (performance degradation)

No long reaches demonstrated so far (record reach $<1 \mathrm{~km}$ )

- Losses so far are well above SMF

only a minor penalty of $\sim 1 \mathrm{~dB}$ after $80 \mathrm{~km}$ of transmission [5]. In the succeeding experiment an optical signal carrying 96 channels x 3 modes x 256 Gb/s DP-16QAM was sent over $119 \mathrm{~km}$ of FMF with the MM-EDFA after $80 \mathrm{~km}$, and successfully demodulated using MIMO-DSP at the receiver side, giving a net data rate of $57.6 \mathrm{~Tb} / \mathrm{s}$ [7]. The main limitation in transmission distance for this particular experiment were the mode MUX and mode DEMUX, adding up to $\sim 20 \mathrm{~dB}$ of loss, which equals around $100 \mathrm{~km}$ of FMF, and caused the transmission system to be limited by amplified spontaneous emission (ASE). Using different mode-multiplex techniques offering a reduced insertion loss like the photonic lantern [8] should directly increase this transmission distance.

In principle even higher-order modulation formats can be mode-MUXed and transmitted over FMF. Fig. 2 shows the curves measured for three mode-MUXing in a back-to-back configuration, which consisted of a mode MUX and mode DEMUX connected with $\sim 10 \mathrm{~m}$ of FMF.

As mentioned before, exploiting the different modes of a FMF will linearly increase the capacity per fiber by the number of modes. But capacity increase using higherorder modulation formats does not seem equally promising because FMFs are still made of glass and the loss therefore is the same as for SMF, still requiring amplification after every $\sim 80 \mathrm{~km}$ which still limits the received OSNR (Table I).

\section{High-CAPACITy PHOtonic BANDgAP FIBER TRANSMISSION SYSTEMS}

A larger increase in capacity per fiber is to be expected if the loss and nonlinear tolerance of fibers can be (greatly) improved. Photonic bandgap fibers (PBGFs), where the light travels in air, offer that prospect [9], i.e. a lower loss and especially a very high nonlinear tolerance, making it possible, in principle, to transmit higher-order modulation formats over long-haul distances. However at the moment the loss is still too high to allow $>10 \mathrm{~km}$ transmission experiments.

Realisation of ultra-low loss PBGFs would allow to increase span lengths thanks to both the lower loss and the increased nonlinear tolerance which would permit higher launch powers. These fibers will inherently be multi-mode as well, offering an improvement in capacity per fiber on three fronts. Using state-of-the art 230 meter of length 19-cell PBGF [10] and only exploiting the fundamental $\left(\mathrm{LP}_{01}\right)$ mode of this fiber direct-detection transmission with low-penalty of $37 \times 40-\mathrm{Gb} / \mathrm{s}$ channels was shown [11]. Recently this capacity was increased 20 -fold by sending for the first time ever coherently detected optically modulated signals over this fiber, $96 \times 250-G b / s$ DP-32QAM [12]. Experiments using the higher order modes as separate transmission lanes were shown in $[13,14]$ with relatively poor performance. By using MIMO-DSP and exploiting the $\mathrm{LP}_{01}, \mathrm{LP}_{11 \mathrm{a}}$ and $\mathrm{LP}_{11 \mathrm{~b}}$ modes of the 19 -cell PBGF, we are now beginning to investigate the potential for high-capacity multi-mode transmission over a PBGF (Fig. 3).

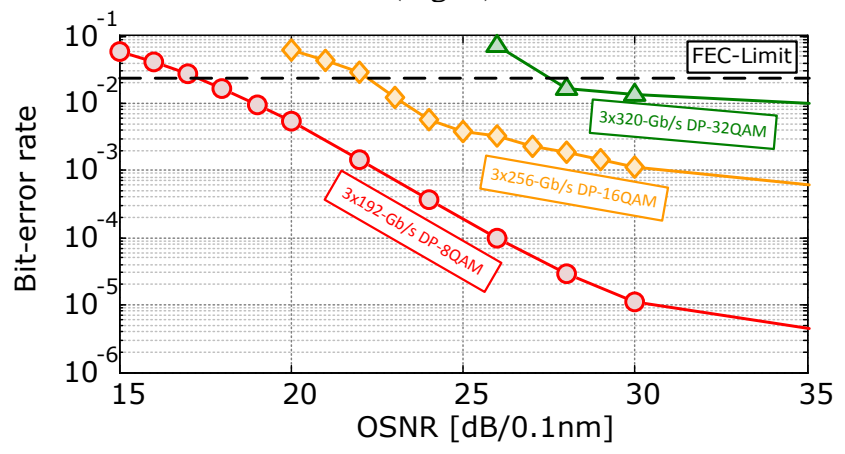

Fig. 3. Average BER after single channel, three mode-multiplexed transmission over $\sim 230 \mathrm{~m}$ of 19 -cell PBGF.

\section{CONCLUSIONS}

We reviewed in this paper the potential of multi-mode solid core fiber and hollow core fiber as a means to increase the capacity per fiber by employing the number of modes as separate transmission lanes and the use of higher-order modulation formats requiring more received OSNR.

\section{Acknowledgement}

This work was supported by the EU FP7-ICT MODE GAP project under grant agreement 258033.

[1] V.A.J.M. Sleiffer et al., Proc. OFC'12, OW4C.3

[2] D. van den Borne et al.,Proc. OECC'11, pp. 429-432

[3] A.D. Ellis, Proc. IEEE Sum. Top. '12, pp. 169-170

[4] R.-J. Essiambre et al., JLT 28, 662-701 (2010)

[5] V.A.J.M. Sleiffer et al., Proc. ECOC'12, TU.1.C.2

[6] E. Ip et. al., Proc. SPIE 8647, 864709 (2013)

[7] V.A.J.M. Sleiffer et al., Opt. Exp. 20, B428-B438 (2012)

[8] R. Ryf et al., Proc. FiO '12, FW6C.4

[9] M.N.Petrovich et al.,Opt. Exp. 16, 4337-4346 (2008)

[10] N. Wheeler et al., Proc. OFC'12, PDP5A.2

[11] R. Slavik et al., ECOC'12, Mo.2.F.2

[12] V.A.J.M. Sleiffer et al., Proc. OFC'13, OW1I.5

[13] J. Carpenter et al., Proc. OFC'12, JW2A.41

[14] J. Xu et al., Optics Express 20, 12449-12456 (2012) 